\title{
Duration distributions for different softness groups of gamma-ray bursts
}

\author{
Y.-P. Qin ${ }^{1,2}$, A. C. Gupta ${ }^{3}$ J. H. Fan ${ }^{1}$, C.-Y. Su ${ }^{4}$, R.-J. Lu ${ }^{2}$
}

\begin{abstract}
Gamma-ray bursts (GRBs) are divided into two classes according to their durations. We investigate if the softness of bursts plays a role in the conventional classification of the objects. We employ the BATSE (Burst and Transient Source Experiment) catalog and analyze the duration distributions of different groups of GRBs associated with distinct softness. Our analysis reveals that the conventional classification of GRBs with the duration of bursts is influenced by the softness of the objects. There exits a bimodality in the duration distribution of GRBs for each group of bursts and the time position of the dip in the bimodality histogram shifts with the softness parameter. Our findings suggest that the conventional classification scheme should be modified by separating the two wellknown populations in different softness groups, which would be more reasonable than doing so with a single sample. According to the relation between the dip position and the softness parameter, we get an empirical function that can roughly set apart the short-hard and long-soft bursts: $S P=(0.100 \pm 0.028) T_{90}^{-(0.85 \pm 0.18)}$, where $S P$ is the softness parameter adopted in this paper.
\end{abstract}

Key words: gamma-rays, bursts — methods, data analysis — methods, statistical PACS: 98.70.Rz, 07.05.Kf, 43.60.Cg, 97.10.Ri

\section{Introduction}

The bimodality in the duration distribution of GRBs suggests that, the GRB events exist in short and long duration classes, which are separated at 2 seconds [1]. In general, the

\footnotetext{
${ }^{1}$ Center for Astrophysics, Guangzhou University, Guangzhou 510006, P. R. China; ypqin@gzhu.edu.cn

${ }^{2}$ Physics Department, Guangxi University, Nanning 530004, P. R. China

${ }^{3}$ Aryabhatta Research Institute of Observational Sciences (ARIES), Manora Peak, Nainital - 263129, India

${ }^{4}$ Department of Physics, Guangdong Industry University, Guangzhou 510006, P. R. China
} 
short duration bursts are harder and long duration bursts are softer. In the hardness ratio vs. duration plot, the two classes are seen to distribute in distinct domains $[1,2]$, where the hardness ratio $H R_{32}$ concerned is defined as the ratio of the total counts in the $100-300 \mathrm{keV}$ and $50-100 \mathrm{keV}$ energy range (later, the hardness ratio is defined as the ratio of the fluence in the $100-300 \mathrm{keV}$ range to the fluence in the $50-100 \mathrm{keV}$ range [3]). When sources of both classes are combined, the hardness ratio is obviously correlated to the duration, while for each of the two classes alone the two quantities are not correlated at all [4]. This in turn strongly suggests the existence of the two classes of GRBs. The study of GRB classification is very important since different classes might have different progenitors. It was proposed that the long duration bursts are caused by the massive star collapsars [5-7] and shot duration bursts are produced in the event of binary neutron star or neutron star-black hole mergers $[8,9]$.

After the successful launch of the Swift satellite [10], a large number of evidences favor the two progenitor proposal for GRBs. Long duration bursts were found to be originated from star-forming regions in galaxies [11], and in some of these, supernovae were detected to accompany the bursts $[12,13]$. On the other hand, short duration bursts were detected in regions with lower star-formation rates, with no evidence of supernovae to accompany them $[14-16]$.

Although GRBs are known to belong to the two distinct classes, the membership of the classes is hard to establish due to the overlap of the duration distributions, which makes the classification of the bursts an unsettled issue. Authors of Ref. [17] showed that, the bimodal distribution of GRBs can be well accounted for by two overlapped lognormal distributions, which suggests that each of the two GRB populations is likely to form a single peak modality and there would be a sufficient number of bursts that are mis-classified by simply applying the criterion $T_{90}=2 s$. Authors of Ref. [18] used a multivariate analysis to discriminate between distinct classes of GRBs, and from the third BATSE catalog they found that instead of two, there are three classes of GRBs, which triggered a debate lasting until the present time.

In distinguishing the X-ray flashes (XRFs), X-ray rich bursts (XRRs) and conventional gamma-ray bursts (CGRBs), authors of Ref. [19] used the ratio between the X-ray fluence $S_{X}$ and the gamma-ray fluence $S_{\gamma}$ as a softness parameter to divide them. Their results seem to be quite satisfactory. We wonder, if the softness parameter can play a role in separating the short-hard and long-soft populations of GRBs from the BATSE catalog. This is the motivation for our analysis given below.

The BATSE catalog has the largest sample of GRBs available until today. We employ this sample in the following analysis since the total number of GRBs in the sample is large enough for a meaningful statistical analysis. 
In Section 2, we investigate if the duration distribution depends on the softness parameter. Based on this investigation, we shortly explore a possible classification of GRBs with an empirical curve in Section 3. The case of Swift is considered in Section 4. Discussion and conclusions are presented in Section 5 .

\section{Duration distributions of GRBs with different softness parameters}

Motivated by Ref. [19]'s work, we wonder if the softness of BATSE bursts can play a role in separating the short-hard and long-soft populations of GRBs. However, most of the BATSE bursts do not have X-ray fluxes, so, we cannot define the softness of the bursts with $S_{X} / S_{\gamma}$. Therefore, we turn to consider the ratio between the fluence in the lowest energy band and that in higher energy bands, as adopted in Ref. [20] to distinguish them. This parameter is no longer applicable to distinguish XRFs, XRRs and CGRBs. Here, we define the softness parameter of BATSE bursts by

$$
S P \equiv \frac{f_{1}}{f_{2}+f_{3}+f_{4}},
$$

where $f_{1}, f_{2}, f_{3}$, and $f_{4}$ are the fluences of the first $(20-50 \mathrm{keV})$, second $(50-100 \mathrm{keV})$, third $(100-300 \mathrm{keV})$, and fourth $(>300 \mathrm{keV})$ BATSE channels, respectively.

As hinted by Ref. [19, 20], we roughly divide the bursts into several groups according to the softness parameter $S P$. To be comparable to the conventional duration distribution plot, we divide the duration range from $0.01 \mathrm{~s}$ to $2000 \mathrm{~s}$ in the logarithm format into 30 bins. We require that the maximum count of each group should be no less than 30 to keep a statistical significance. For the softest group, we require that if there exist some short duration bursts in this group, the corresponding count should be noticeable. In this way, we get four groups of bursts. The distributions of durations $\left(T_{90}\right)$ of these groups are displayed in Figure 1 , where $T_{90}$ of a burst is the time interval during which $90 \%$ of the total observed counts have been detected. We find that the duration histogram of each group bears a bimodality. The positions of the dip lying between the two peaks of the bimodality of different groups differ significantly, where the softer the group, the smaller value of the duration position of the dip. If separating the bursts into short and long duration classes, then in different ranges of the softness parameter the classes occupy significantly different percentage of the total counts. The figure shows that the long duration bursts occupy a very large percentage of the softest population $(S P \geq 0.1$ ), and they cover a large range of durations, starting from $\sim 1$ s to $\sim 1000$ s. In contrast, short duration bursts occupy a very small percentage of the softest population, and they cover a very small range of durations. The situation changes for harder groups: the percentage of long duration bursts becomes smaller and they cover 


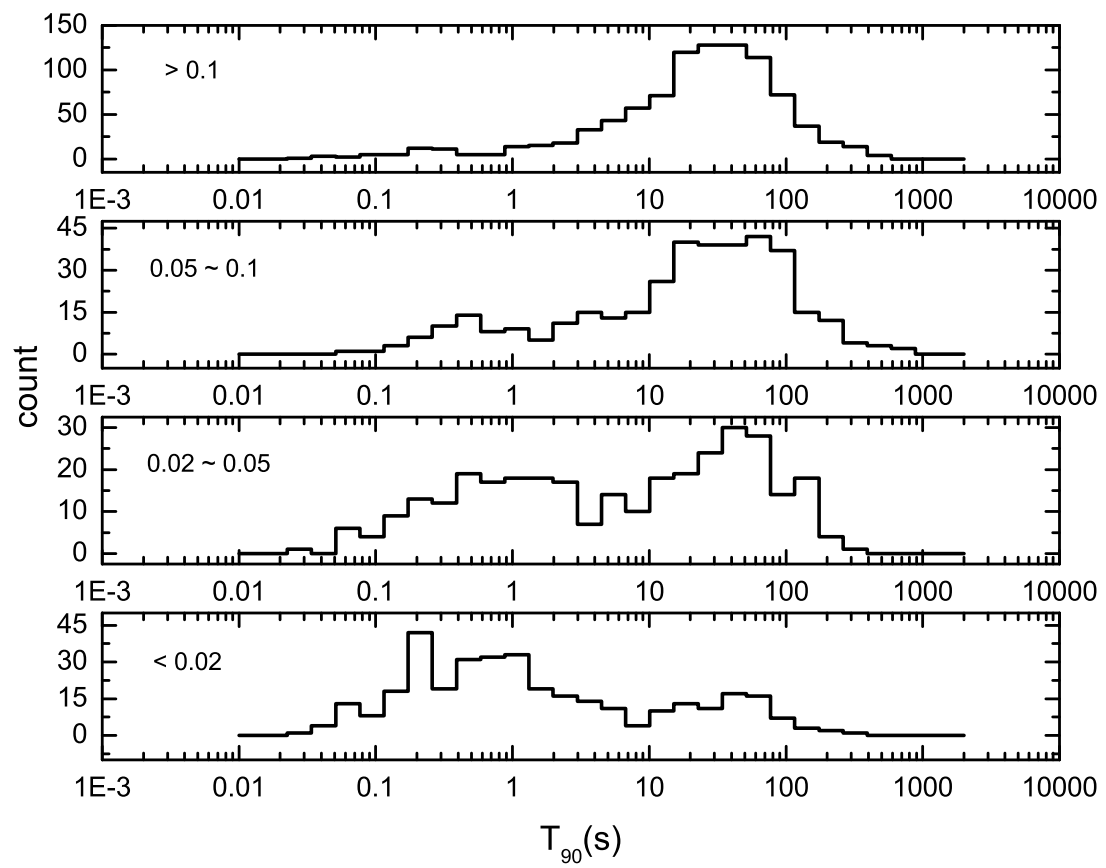

Fig. 1.- Duration distributions of the BATSE bursts with different softness parameters. Range of the adopted softness parameter is mentioned at the upper left corner for each panel. 
smaller ranges of durations, while the percentage of short duration bursts becomes larger and they cover larger range of durations.

In the conventional classification of GRBs, a dip around $2 \mathrm{~s}$ in the duration histogram plot in Figure 1a of Ref. [1] was proposed. For a more precise measurement, the authors fitted a quadratic function between the two peaks in the histogram and determined its minimum to be $T_{90}=(1.2 \pm 0.4) s$. As an empirical analysis, we adopt this quadratic function fitting method to locate the dip position from the plots in Figure 1. In Table 1, dip positions and the numbers of long and short duration bursts divided by this feature for various softness groups are listed. In the first column, the softness parameter range of each group is presented. The mean of the softness parameter calculated with all the individual $S P$ values of the bursts of the corresponding group is presented in the second column. Positions of the dip are given in the third column. The numbers of short and long duration bursts separated by the dip are presented in the fourth and fifth columns respectively.

\section{Empirical curve roughly setting apart two populations of BATSE bursts}

A relation between the softness parameter and the dip position of the bi-modal duration distribution of BATSE bursts hinted by Figure 1 can be evaluated from the data of Table 1. In Figure 2, we display the result of a power-law analysis on the data. Fitting the data with a power-law function yields (by performing a Spearman correlation analysis with the ORIGIN software):

$$
S P=(0.100 \pm 0.028) T_{90}^{-(0.85 \pm 0.18)} .
$$

The following are the statistical results of a linear correlation analysis between $\log S P$ and $\log T_{90}$ : the correlation coefficient, -0.958 ; the number of data points, 4 ; the probability of rejecting the null hypotheses, 0.0417 . Owing to the limit of the total counts, we have only 4 data points in Figure 2. The statistical analysis performed here is thus not robust (we hence regard this fitting curve as an empirical function - see the statement below). Note that one can divide the BATSE catalog into more groups to have more data points. As a result, one might find difficulties to locate the dip suggested in Figure 1 due to data fluctuations.

In Figure 3, we display the plot of the softness parameter vs. the duration of BATSE bursts, where the function of Equation (2) is also plotted. The figure shows that there exist two populations of bursts which are clustered in two distinct domains in the plot. Between the two clusters there is a "gap" with relatively sparse counts. The function of Equation (2), which is the fitting curve in Figure 2, is seen to pass through the "gap", roughly separating the two populations. As the figure shows, most sources of the population below the fitting curve are the conventional short bursts (the duration is smaller than $2 \mathrm{~s}$ ), and most sources 
Table 1. Parameters of different softness groups.

\begin{tabular}{lllll}
\hline \hline & \multicolumn{1}{c}{$\overline{S P}$} & $T_{90}(s)$ & short $^{a}$ & long $^{b}$ \\
\hline $0.1 \leq S P$ & $0.28 \pm 0.24$ & $0.52_{-0.42}^{+0.65}$ & 41 & 895 \\
$0.05 \leq S P<0.1$ & $0.073 \pm 0.014$ & $0.82_{-0.44}^{+1.52}$ & 42 & 328 \\
$0.02 \leq S P<0.05$ & $0.0338 \pm 0.0086$ & $3.3_{-2.0}^{+5.2}$ & 135 & 186 \\
$S P<0.02$ & $0.0103 \pm 0.0056$ & $16.2_{-3.2}^{+81.9}$ & 278 & 67 \\
\hline
\end{tabular}

${ }^{a}$ The number of sort duration bursts with their duration being no less than the $T_{90}$ value presented in the third column.

bThe number of long duration bursts with their duration being larger than the $T_{90}$ value presented in the third column.

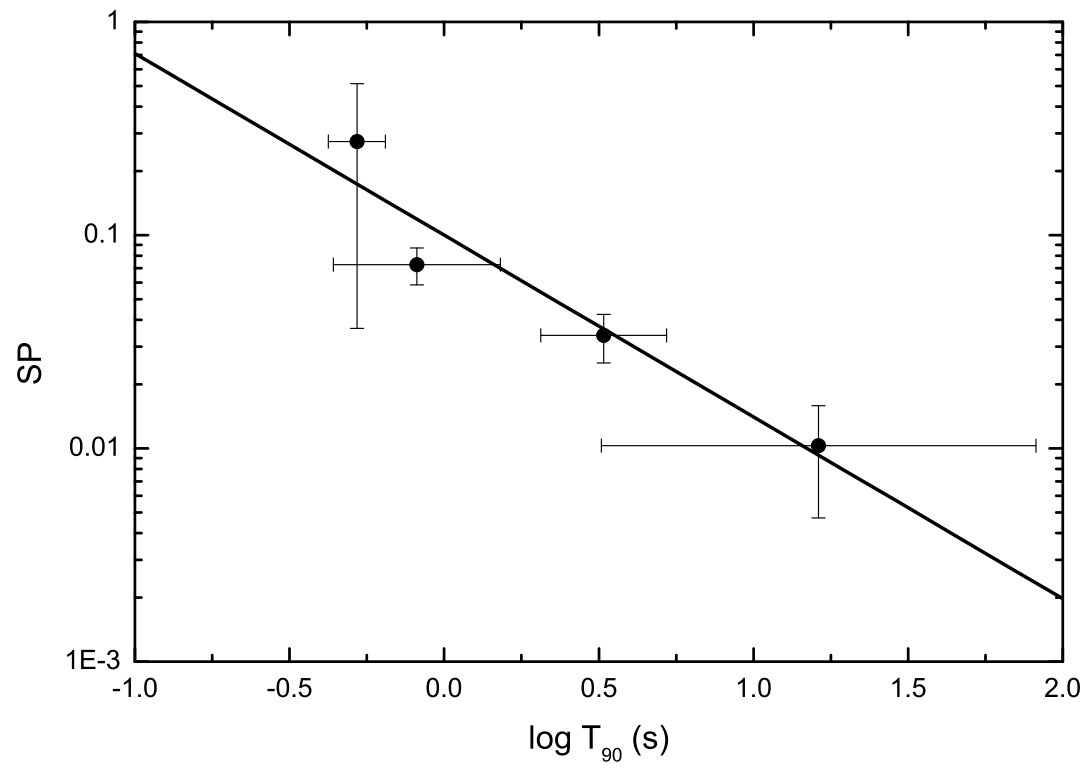

Fig. 2.- Relation between the softness parameter and the position of the duration distribution dip drawn from the BATSE catalog. 


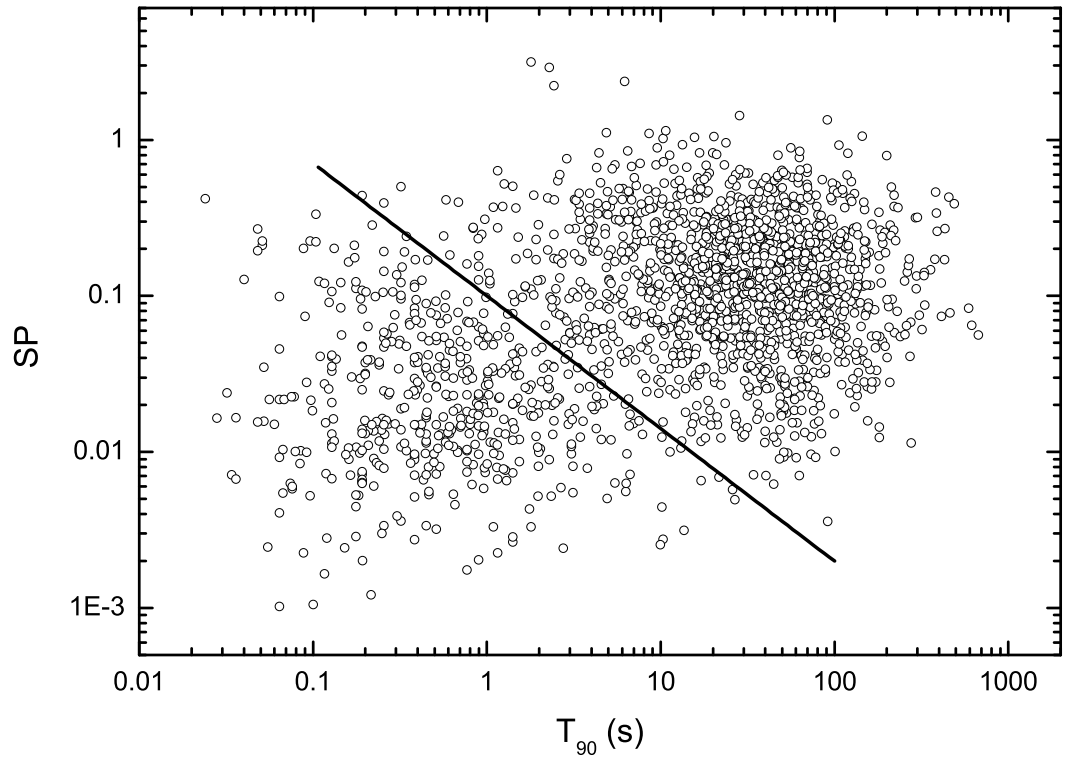

Fig. 3.- Plot of the softness parameter vs. the duration of BATSE bursts, where the solid line is the function of Equation (2). 


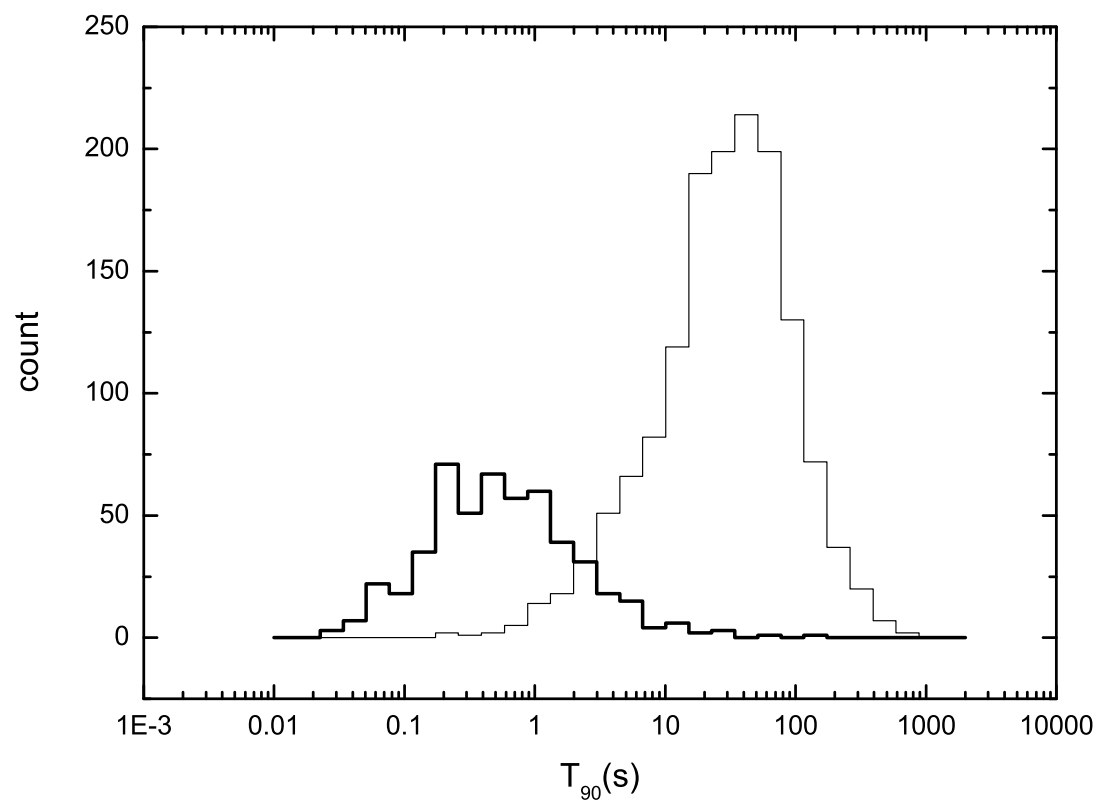

Fig. 4.- Duration distributions of the two populations divided by the curve of Equation (2), where the thick line represents the short-hard population while the thin line denotes the long-soft population drawn from the BATSE catalogue.

of the population above the fitting curve are the conventional long bursts (the duration is larger than $2 \mathrm{~s})$.

We find that: a) the number of the population below the fitting curve is 511, and among them there are 433 bursts ( $84.7 \%$ of the population) with their duration satisfying $T_{90} \leq 2 s$; b) the number of the population above the fitting curve is 1461 , and among them there are 1418 bursts (97.1\% of the population) with their duration satisfying $T_{90}>2 s$. The duration distributions of the two populations divided by the fitting curve is shown in Figure 4. Remind that the fitting curve represents the relation between the dip positions of the duration distribution of bursts and the softness parameter. According to this analysis (refer to Figures 1, 3 and 4), we regard the fitting curve as an empirical curve roughly setting apart the short-hard and long-soft populations of BATSE GRBs in the softness vs. duration plot. 


\section{In the case of Swift}

GRBs observed by Swift are becoming more and more important due to the excellent ability of Swift to detect the afterglows of the objects. The number of bursts collected by Swift has been steadily growing up since the launch of the satellite [10]. Would the statistical analysis performed above be applicable to the current Swift data set?

To answer this question, we search in literature and collect the Swift data which contain the values of the duration and the four Swift channel fluences. The data are available in Ref. [21] where the first Swift BAT GRB catalog is presented. From Ref. [21] we get 222 bursts with their $T_{90}$ as well as $f_{1}(15-25 \mathrm{keV}), f_{2}(25-50 \mathrm{keV}), f_{3}(50-100 \mathrm{keV})$, and $f_{4}(100-150 \mathrm{keV})$ being available. Compared with Table 1 we find that this number of bursts is too small to be used to perform the same analysis. This explains why we focus our attention on the BATSE catalog instead of the Swift catalog.

Even though the number is so small, we manage to divide the bursts into two groups according to their softness parameters to check their duration distributions. The same definition of the softness parameter is adopted, although the energy channels of Swift are not the same as those of BATSE (also, $T_{90}$ would not be exactly the same as that measured in BATSE, since the energy range of bursts would play a role in determining the quantity). Those Swift bursts with $S P \geq 0.1$ are included in the softer group, whilst the others belong to the harder group. Duration distributions of the two groups are shown in Figure 5 . We observe that, although the statistical significance is much less than that in the BATSE catalog due to the small number of bursts, the same trend is observed in the figure: the duration histogram of each group is likely to bear a bimodality; the positions of the dip lying between the two peaks of the bimodality of the two groups are different, where the softer the group, the smaller value of the duration position of the dip; when separating the bursts of each group into short and long duration classes according to the apparent dip, then any of the two classes occupies significantly different percentage of the total count in different groups, e.g., the softer the group, the larger percentage of the long duration bursts. Comparing Figure 1 with Figure 5 one can find that the adopted statistical analysis is hard to be applied to the current Swift data due to the limited number of bursts.

To check the trend in other ways, let us ignore the statistical significance and fit a quadratic function between the two peaks in the histogram of each panel of Figure 5 and determine the minimum of the curve as the position of the dip. The result is displayed in Figure 6, where as a comparison, the BATSE result shown in Figure 2 is also presented. The same trend of the empirical function is maintained if we rely on the two Swift data points in the figure. However, we would like to emphasize that one should not take the two data points so serious since the numbers adopted in Figure 5 are quite small so that the statistical 


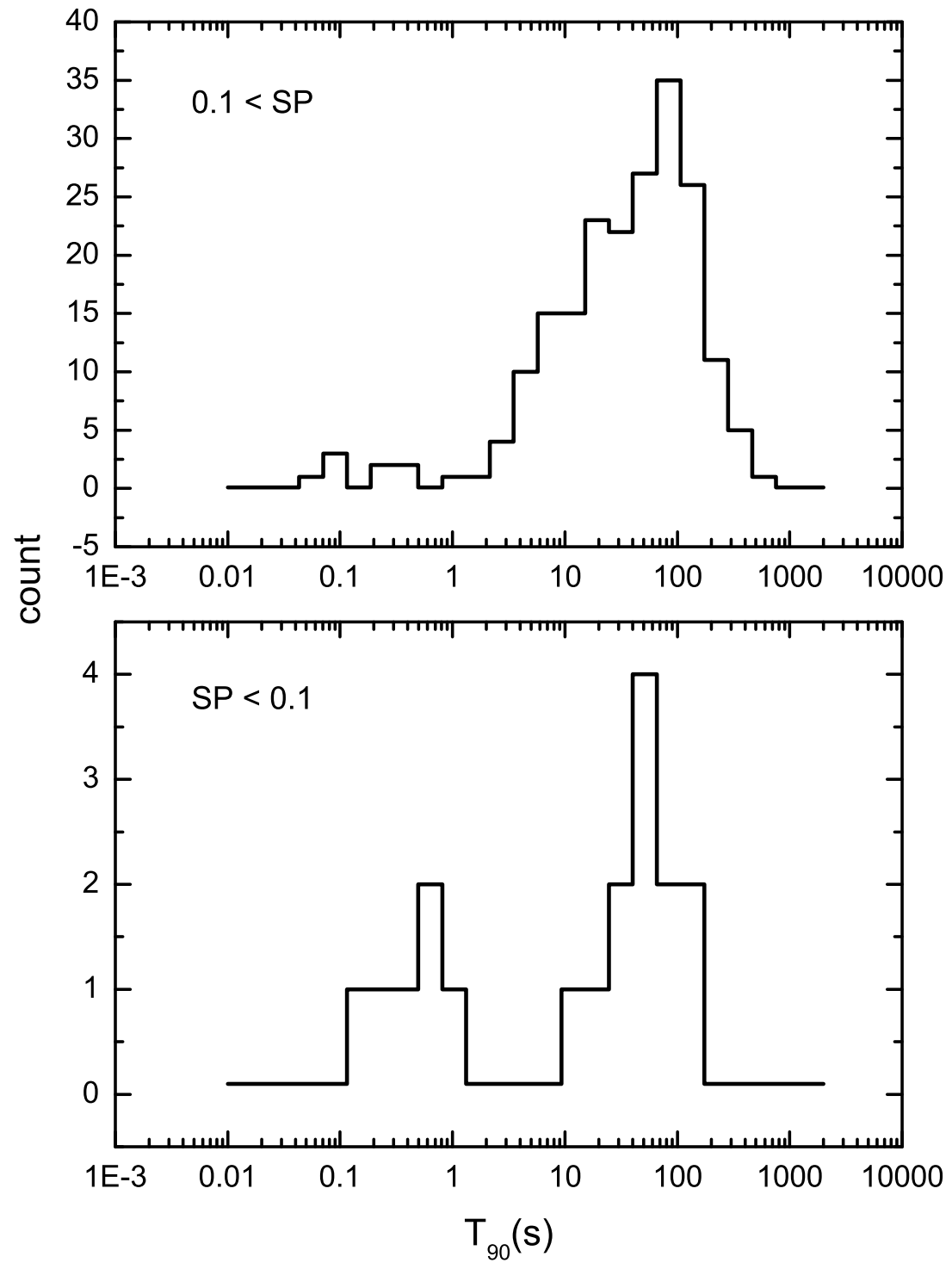

Fig. 5.- Duration distributions of Swift bursts with different softness parameters. The range of the adopted softness parameter is mentioned at the upper left corner of each panel. 


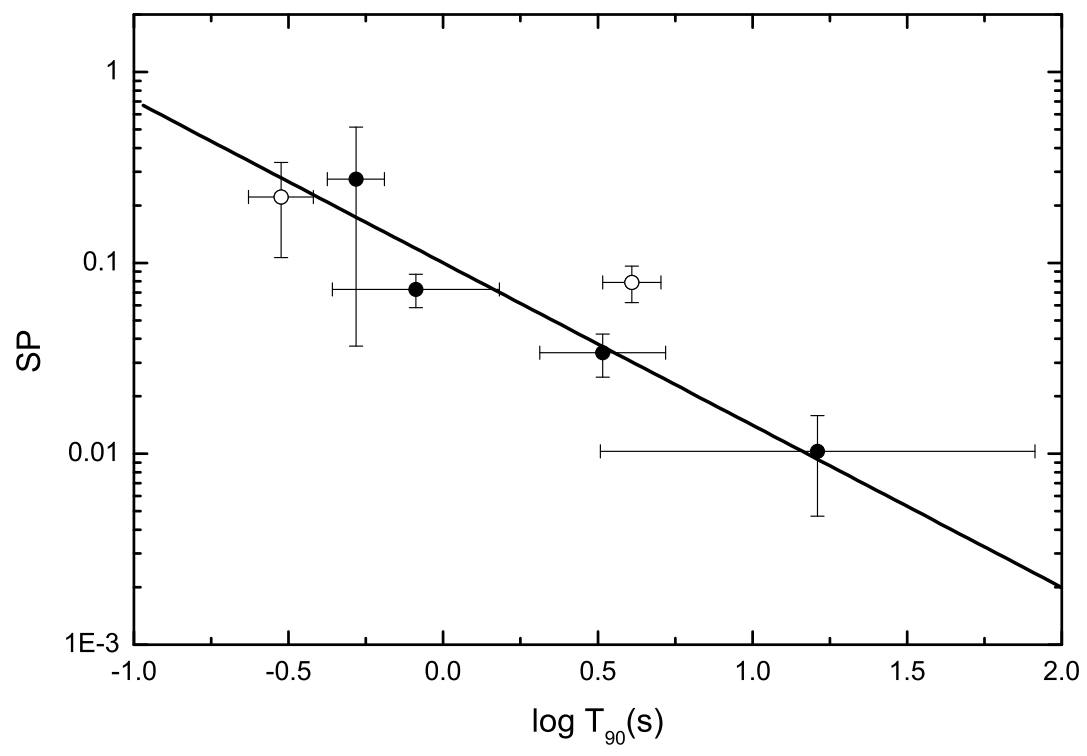

Fig. 6.- Relation between the softness parameter and the position of the duration distribution dip drawn from the Swift catalog (open circles). Other symbols are the same as they are in Figgure 2.

result is not so robust. In addition, we adopt the same definition of the softness parameter to analyze the BATSE and Swift catalogs but this definition would correspond to at least a slightly different quantities since the energy ranges of the corresponding channels are not the same. While this is not an unsolvable problem currently, but the number of bursts is. We hope that the growing Swift burst number would provide a reliable analysis in the near future.

\section{Discussion and conclusions}

Motivated by Ref. [19]'s work, we investigate if the softness of bursts plays a role in the conventional classification of GRBs. We employ the BATSE catalog and define the softness parameter as the ratio of the fluence in the first channel to the sum of the fluences in the second, third and fourth channels. The duration distributions of different groups of BATSE GRBs associated with distinct softness parameters are explored. From the analysis we get 


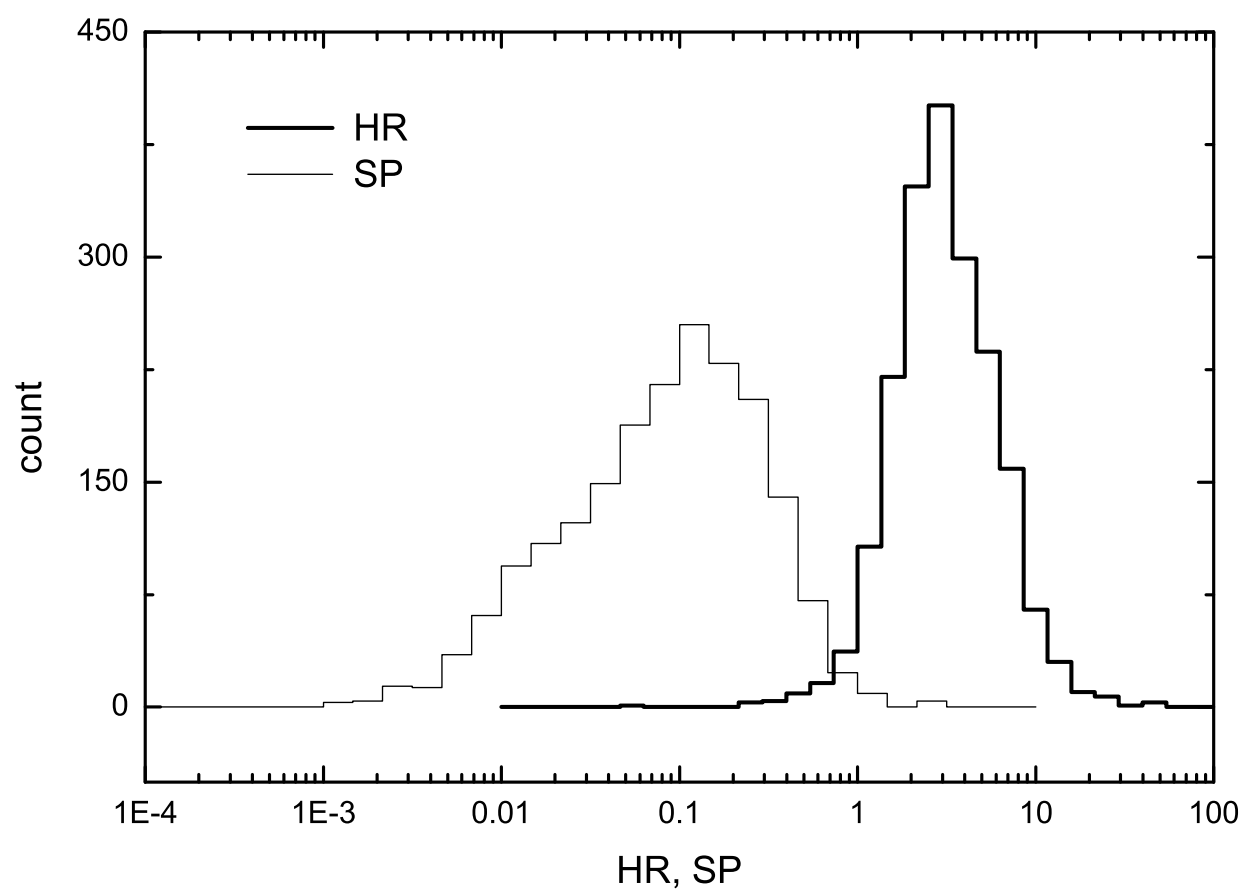

Fig. 7.- Distributions of the conventional hardness ratio (the thick line) and the softness parameter (the thin line) of the bursts in the BATSE catalog.

an empirical curve that can roughly set apart short and long duration bursts. The analysis shows that the conventional classification of GRBs with the duration of bursts is influenced by the softness of the objects.

As is known, the hardness ratio is an active factor to tell the spectral difference between the short and long duration bursts of GRBs. Why do we adopt the softness parameter instead of the conventional hardness ratio to investigate the issue? As mentioned above, it is Ref. [19]'s work on dividing XRFs, XRRs, and CGRBs with another softness parameter $\left(S_{X} / S_{\gamma}\right)$ that motivates us for the exploration of the softness parameter as a possible factor active in the classification of GRBs. While the role of the conventional hardness ratio in GRB classification has been well studied for a long time, the role of the softness parameter has not been sufficiently explored. Another reason for doing so is illustrated in Figure 7, where distributions of both the conventional hardness ratio and the softness parameter are presented. We find that, while the conventional hardness ratio spans about one magnitude interval, the softness parameter covers approximately two magnitude range. This suggests that, if both the conventional hardness ratio and the softness parameter can be adopted as a parameter to distinguish different types of burst, then the latter must be more sensitive 
than the former, since the difference of the latter is more easily noticeable.

What would one get in the conventional hardness ratio vs. duration plot, if the bursts of the BATSE catalog are divided by the empirical curve, the function of Equation (2)? The result is shown in Figure 8 where we find that two distinct populations are clustered in the conventional domains of the short and long duration classes. The well-known correlation properties between the hardness ratio and duration of the two classical populations are maintained. That is, for any of the two populations, the hardness ratio is not at all correlated with the duration, while the two elements are obviously correlated when the two populations are combined. This is not surprising, since $84.7 \%$ bursts of the population below the fitting curve are the conventional short duration bursts $\left(T_{90} \leq 2 s\right)$, and $97.1 \%$ bursts of the population above the fitting curve are the conventional long duration bursts $\left(T_{90}>2 s\right)$. This together with Figure 4 show that dividing BATSE bursts with the empirical curve or with the duration criterion $T_{90}=2 s$ would not give rise to a statistical difference (or, the statistical properties of the two resulting populations would not be significantly different for the two criterions). This in turn suggests that the modification to the conventional method (e.g., by considering different softness groups instead of a single sample, or by adopting the empirical curve instead of the $T_{90}=2 s$ criterion) would not lead to a noticeable physical discrepancy between the two populations.

As Figure 8 shows, the two populations divided by the empirical curve heavily overlap in the hardness ratio vs. duration plot. This together with the overlapping in Figures 3 and 4 imply that, the two well-known populations are unlikely to be sharply separated merely by quantities such as the duration, the hardness ratio and the softness parameter, or the relations between them. To distinguish the intrinsic short-hard and long-soft populations, other supplemental criterions are needed. Among them, the most desirable one is the location of bursts in the host galaxies, as observable in some Swift bursts.

One might notice that the softness parameter adopted here is in fact an inverted BATSE gamma-ray hardness ratio $H R_{234 / 1} \equiv\left(f_{2}+f_{3}+f_{4}\right) / f_{1}$. Therefore, the fact that the dip existed in the bimodality in the duration distribution of GRBs shifts with the softness parameter (see Figure 1) is equivalent to the fact that the dip position shifts with the hardness ratio $H R_{234 / 1}$.

What is the difference between our analysis and the conventional one? The conventional investigation considers a single sample from a catalog whilst our study considers different groups of softness from the same catalog. The conventional one yields a single dip position of the histogram of the duration distribution while our analysis produces several dip positions which are found to vary with the softness. The statistical methods adopted to distinguish the long and short duration classes in both cases are exactly the same, but the results 

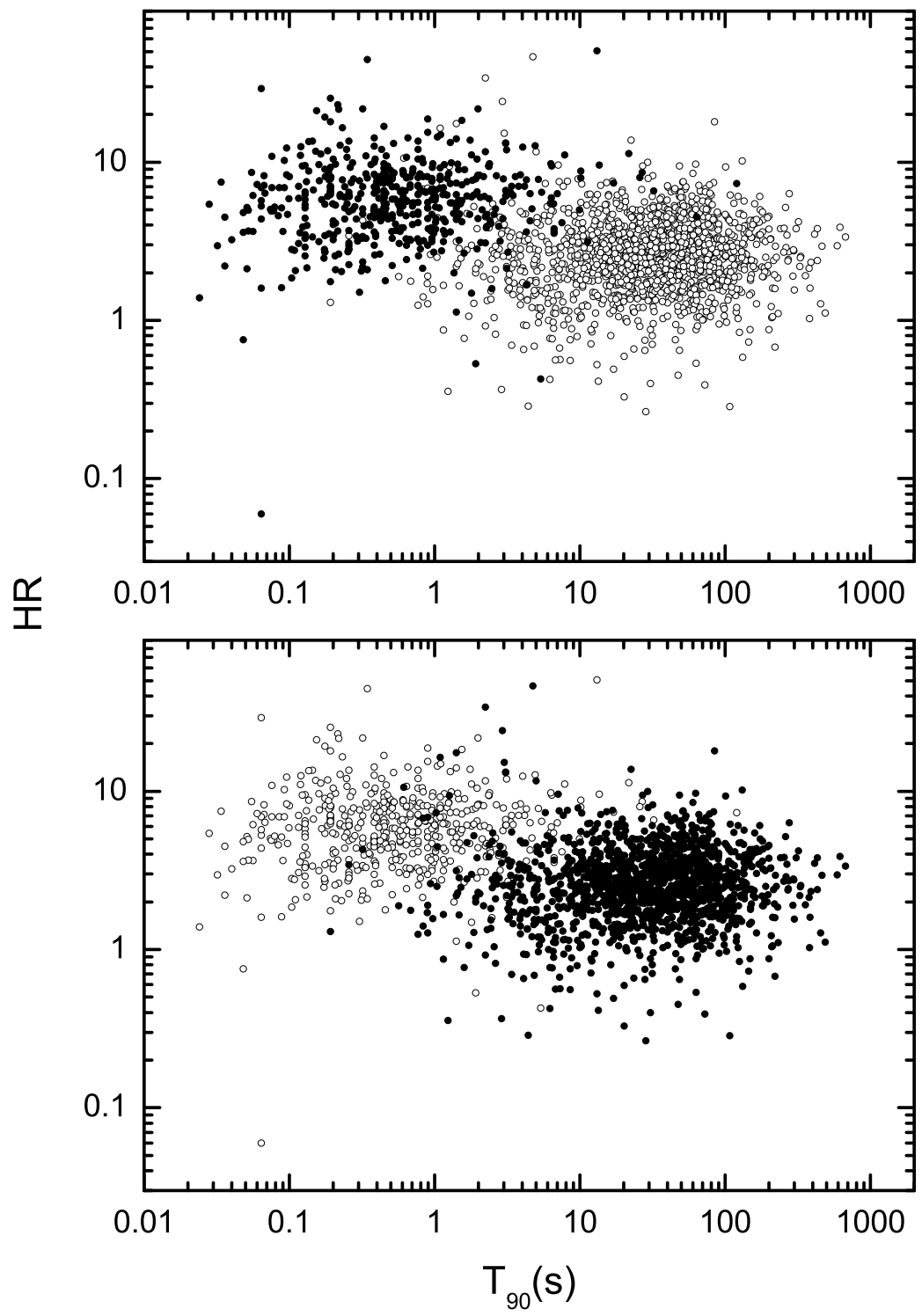

Fig. 8. - Hardness ratio vs. duration plot for the short duration bursts (filled circles in the upper panel; open circles in the lower panel) and long duration bursts (filled circles in the lower panel; open circles in the upper panel) divided by the function of Equation (2) from the BATSE catalog. 
are different. Our analysis suggests that the conventional classification should be modified by setting apart the two populations in different softness groups, which would be more reasonable than doing this with a single sample. Another possible modification is to separate the two populations in the softness vs. duration plot with an empirical function as long as the function is reliable enough (when the total number of bursts is much larger than the current available one, the function so obtained would be more robust in terms of statistics, e.g., in that case one would get more data points rather than only 4 in Figure 2 under the same requirements).

Assume that we take the empirical function as the criterion to classify GRBs. If so, is the new classification statistically better than the old one? As discussed above, as much as $84.7 \%$ of the newly classified short-hard bursts are the conventional short duration bursts and as much as $97.1 \%$ of the newly classified long-soft bursts are the conventional long duration bursts. The new method is only a modification to the conventional one but not a totally different one. Therefore we cannot expect an entire difference between bursts identified by the two kinds of classification. However, if we believe that each of the two populations follows a lognormal distribution of the duration of bursts, as suggested in Ref. [17], then we can check the statistical difference of the two classifications in this light. Presented in Figure 9 is the fitting result of a lognormal distribution curve to the duration distributions of BATSE bursts for the two classifications. The statistical improvement from the new method is obviously observable.

As Figure 9 shows, compared with the short duration class, the long duration class is more poorly fitted by a lognormal distribution. This indicates that if the empirical function is used as a criterion to separate the BATSE bursts, the resulting long-soft population might contain a "third" subclass. As mentioned above, about one decade ago, authors of Ref. [18] used a multivariate analysis to discriminate between distinct classes of GRBs and found that there exist three classes of GRBs instead of only two. The debate of the existence of the "third class" has then been triggered and lasting until the present time [22-28]. We suspect, if a detailed analysis based on what is shown in Figure 9 is helpful in ending the debate, and hence hope to see such an investigation in the near future (for example, to explore a fit to the duration distribution of the long-soft population with the method of the superposition of two lognormal distributions, as that adopted in Ref. [25]).

What result would a different number of intervals of the softness parameter produce? Obviously, larger intervals would lead to a smaller number (smaller than 4) of data points in Figure 2, which will not be considered here. As mentioned previously, smaller intervals would give rise to more data points in the figure, but the statistical significance will be harmed. For example, when we divide the BATSE catalog into 7 instead of 4 groups, the 

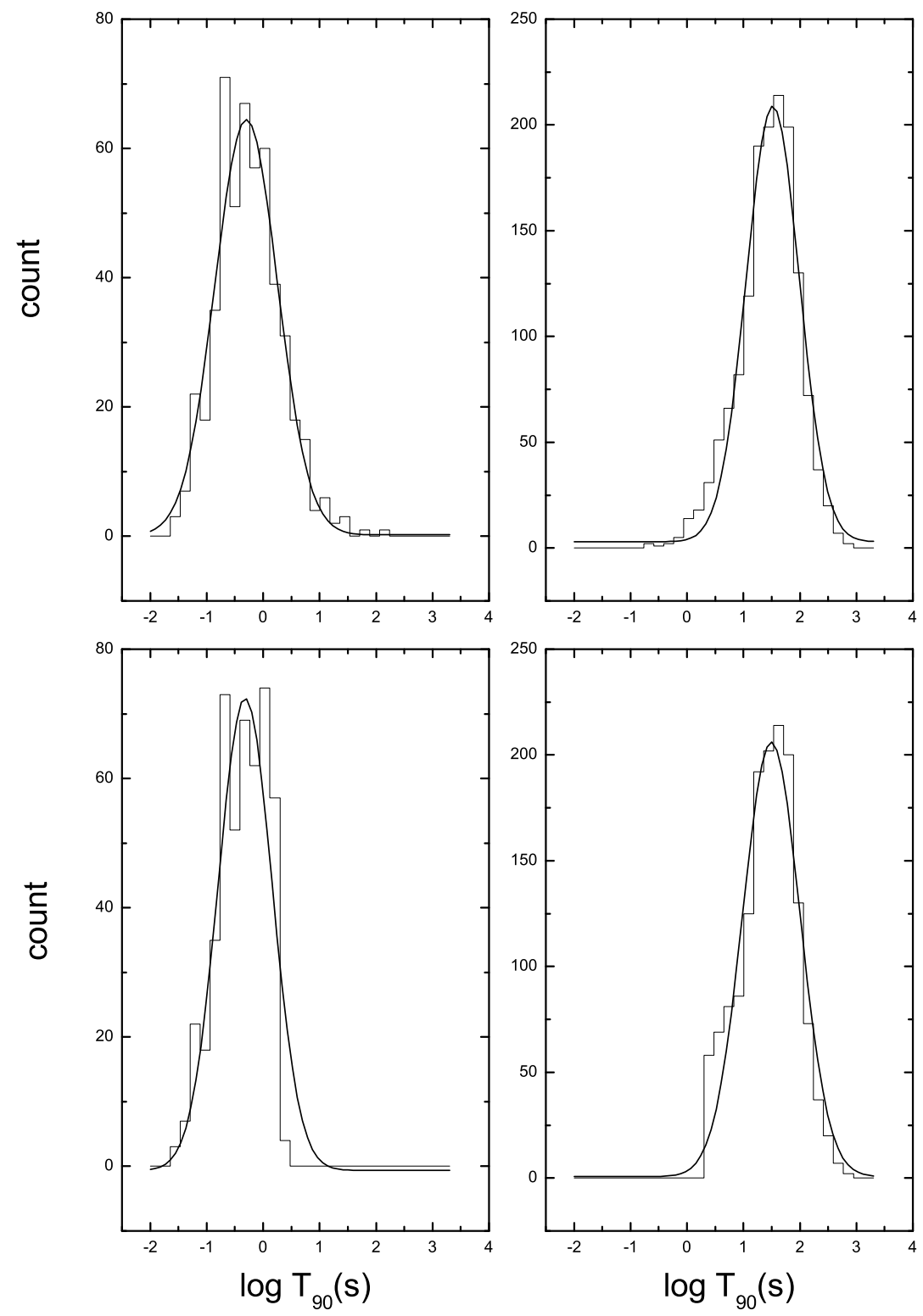

Fig. 9.- Duration distributions (the thin line) of BATSE bursts fitted by a lognormal distribution curve (the thick line) for the conventional short (the left bottom panel; the reduced chi-square for the fit is 85.6) and long (the right bottom panel; the reduced chisquare for the fit is 305) duration classes, and for the newly classified short-hard (the left top panel; the reduced chi-square for the fit is 35.8) and long-soft (the right top panel; the reduced chi-square for the fit is 227) populations by the empirical function of Equation (2), respectively. 


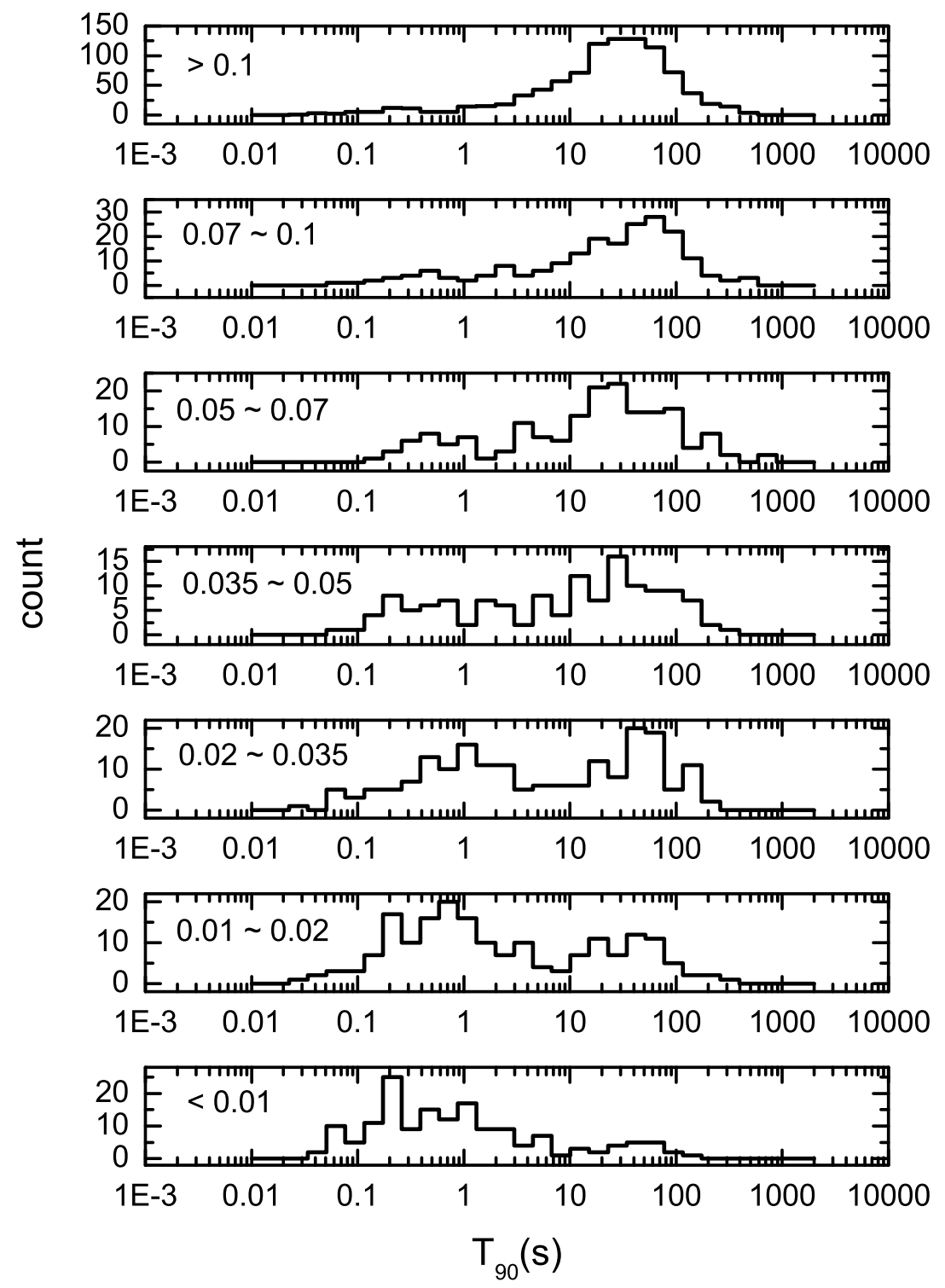

Fig. 10.- Duration distributions of seven groups of the BATSE bursts divided according to the softness parameters. Range of the adopted softness parameter is mentioned at the upper left corner of each panel. 
requirement that the maximum count of each group should be no less than 30 would no longer be satisfied (this is the reason why we prefer to divide the BATSE catalog into 4 groups). As a result, more obvious fluctuation of data would be expected. The result is shown in Figure 10. Compared with Figure 1 we find that data fluctuation is indeed more obvious in Figure 10. Following the same method, we get an empirical curve from Figure 10 , which is $S P=(0.104 \pm 0.019) T_{90}^{-(0.95 \pm 0.11)}$. Parameters of this curve are in agreement with Equation (2) (within the error bars, values of the fitting parameters obtained in both cases are the same), indicating that our analysis does not rely strongly on the choice of the number of intervals of the softness parameter.

According to the above analysis, we reach the following conclusions. a) When dividing BATSE bursts into several groups according to their softness parameters, the duration distribution of each group bears a bimodality histogram. b) Between the two peaks of the bimodality exhibits a feature of dip that sets apart two subgroups, shorter duration subgroup and longer duration subgroup. c) The dip position shifts with the softness parameter, where the softer the group, the smaller value of the duration position of the dip. d) For groups with different softness parameters, the short duration subgroup occupy significantly different percentage of the total count, where the softer the group, the smaller percentage of the total count it occupies (for the long duration subgroup, the conclusion is the opposite). e) Deduced from the relation between the dip position and the softness parameter we find an empirical function that can roughly separate the short-hard and long-soft populations.

As a primary investigation, we find a statistical difference between the duration distributions of a single sample and multi-group samples from the BATSE catalog. We do not know what causes this difference. Nor are there any models that have ever predicted this. Lacking the knowledge of the causing, the physical interpretation associated with the proposed modification of the classification is currently unavailable. In the same way, the phenomenon of overlapping is currently not able to be explained. Other independent investigations are needed to understand the new findings as well as the overlapping phenomenon.

This work is supported in part by the National Natural Scientific Foundation of China (10633010, 10573005, 10747001) and the 973 project (No. 2007CB815405). We also thank the financial support from the Guangzhou Education Bureau and Guangzhou Science and Technology Bureau.

1 Kouveliotou C, Meegan C A, Fishman G J, et al. Identification of two classes of gamma-ray bursts. Astrophys J, 1993, 413(2): L101-L104

2 Fishman G J, Meegan C A. Gamma-Ray Bursts. Annu Rev Astron Astrophys, 1995, 
33: $415-458$

3 Paciesas W S, Meegan C A, Pendleton G N, et al. The Fourth BATSE Gamma-Ray Burst Catalog (Revised). Astrophys J Suppl Ser, 1999, 122(2): 465-495

4 Qin Y-P, Xie G-Z, Xue S-J, et al. The Hardness-Duration Correlation in the Two Classes of Gamma-Ray Bursts. Publ Astron Soc Japan, 2000, 52: 759-761

5 Woosley S E. Gamma-ray bursts from stellar mass accretion disks around black holes. Astrophys J, 1993, 405(1): 273-277

6 Paczynski B. Are Gamma-Ray Bursts in Star-Forming Regions? Astrophys J, 1998, 494: L45-L48

7 MacFadyen A I, Woosley S E. Collapsars: Gamma-Ray Bursts and Explosions in "Failed Supernovae". Astrophys J, 1999, 524(1): 262-289

8 Paczynski B. Gamma-ray bursters at cosmological distances. Astrophys J, 1986, 308: L43-L46

9 Eichler D, Livio M, Piran T, et al. Nucleosynthesis, neutrino bursts and gamma-rays from coalescing neutron stars. Nature, 1989, 340: 126-128

10 Gehrels N, Chincarini G, Giommi P, et al. The Swift Gamma-Ray Burst Mission. Astrophys J, 2004, 611(2): 1005-1020

11 Fruchter A S, Levan A J, Strolger L, et al. Long $\gamma$-ray bursts and core-collapse supernovae have different environments. Nature, 2006, 441(7092): 463-468

12 Stanek K Z, Matheson T, Garnavich P M, et al. Spectroscopic Discovery of the Supernova 2003dh Associated with GRB 030329. Astrophys J, 2003, 591(1): L17-L20

13 Hjorth J, Sollerman J, Moller P, et al. A very energetic supernova associated with the $\gamma$-ray burst of 29 March 2003. Nature, 2003, 423(6942): 847-850

14 Barthelmy S D, Chincarini G, Burrows D N, et al. An origin for short $\gamma$-ray bursts unassociated with current star formation. Nature, 2005, 438(7070): 994-996

15 Berger E, Price P A, Cenko S B, et al. The afterglow and elliptical host galaxy of the short $\gamma$-ray burst GRB 050724. Nature, 2005, 438(7070): 988-990

16 Hjorth J, Watson D, Fynbo J P U, et al. The optical afterglow of the short $\gamma$-ray burst GRB 050709. Nature, 2005, 437(7060): 859-861

17 McBreen B, Hurley K J, Long R, et al. Lognormal Distributions in Gamma-Ray Bursts and Cosmic Lightning. Mon Not R Astron Soc, 1994, 271: 662-666 
18 Mukherjee S, Feigelson E D, Jogesh Babu G, et al. Three Types of Gamma-Ray Bursts. Astrophys J, 1998, 508(1): 314-327

19 Sakamoto T, Lamb D Q, Kawai N, et al. Global Characteristics of X-Ray Flashes and X-Ray-Rich Gamma-Ray Bursts Observed by HETE-2. Astrophys J, 2005, 629(1): 311-327

20 Sakamoto T, Hullinger D, Sato G, et al. Global Properties of X-Ray Flashes and X-Ray-Rich Gamma-Ray Bursts Observed by Swift. Astrophys J, 2008, 679(1): 570-586

21 Sakamoto T, Barthelmy S D, Barbier L, et al. The First Swift BAT Gamma-Ray Burst Catalog. Astrophys J Suppl Ser, 2008, 175(1): 179-190

22 Horvth I A. Third Class of Gamma-Ray Bursts? Astrophys J, 1998, 508(2): 757-759

23 Hakkila J, Haglin D J, Pendleton G N, et al. Gamma-Ray Burst Class Properties. Astrophys J, 2000, 538(1): 165-180

24 Balastegui A, Ruiz-Lapuente P, Canal R. Reclassification of gamma-ray bursts. Mon Not R Astron Soc, 2001, 328(1): 283-290

25 Horvth I. A further study of the BATSE Gamma-Ray Burst duration distribution. Astron Astrophys, 2002, 392: 791-793

26 Rajaniemi H J, Mahonen P. Classifying Gamma-Ray Bursts using Self-organizing Maps. Astrophys J, 2002, 566(1): 202-209

27 Chattopadhyay T, Misra R, Chattopadhyay A K, et al. Statistical Evidence for Three Classes of Gamma-Ray Bursts. Astrophys J, 2007, 667(2): 1017-1023

28 Henry J P. A Measurement of the Density Parameter Derived from the Evolution of Cluster X-Ray Temperatures. Astrophys J, 1997, 489: L1-L5 